Onkologe 2019 25 (Suppl 1):S77-S82 https://doi.org/10.1007/s00761-019-0609-y Online publiziert: 12. Juni 2019

(c) Der/die Autor(en) 2019

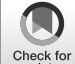

Daniel Heudobler - Wolfgang Herr - Simone Thomas

Klinik und Poliklinik für Innere Medizin III, Universitätsklinikum Regensburg, Regensburg, Deutschland

\title{
Immuntherapien in der Hämatologie und Onkologie
}

einer Immunaktivierung eine überschießende Immunantwort einzudämmen, kommt es auf Ziel- und Immunzellen zur Hochregulation von antiinflammatorischen Kontrollproteinen (sog. Checkpoints), die sich ebenfalls Tumoren $\mathrm{zu}$ Nutze machen, um einer Immunantwort zu entgehen.

\section{Immuncheckpointinhibitoren}

Immuncheckpoints wirken einer überschießenden Immunantwort entgegen. Klinisch sind hierbei 2 Signalwege von besonderer Bedeutung, deren Proteine von T-Zellen exprimiert werden:

- das zytotoxische T-Lymphozytenassoziierte Antigen 4 (CTLA-4),

- das Programmierter-Zelltod-1(PD1)-Molekül.

\section{Grundlagen der modernen Immunonkologie}

Das Immunsystem besitzt die Fähigkeit, spezifische Zielstrukturen zu erkennen und zwischen selbst und fremd zu unterscheiden. Darüber hinaus können seine einzelnen Bestandteile miteinander kommunizieren, Reaktionen regulieren sowie sich erinnern.

\section{》) Zielstrukturen der Immunabwehr sind kurze Proteinbruchstücke oder Zelloberflächenproteine}

Zielstrukturen der Immunabwehr sind entweder kurze Proteinbruchstücke (Peptide), die von den humanen Leukozytenantigenen (HLA) an T-Zellen präsentiert werden oder Zelloberflächenproteine, die von B-Zellen oder Antikörpern erkannt werden. Um nach

\section{Zugelassene Immun- checkpointinhibitoren}

Ipilimumab (anti-CTLA-4) war der erste ICPi, der klinisch einen Benefit zeigte und das mediane Überleben (mOS) bei Patienten mit metastasiertem Melanom von 6 Monaten im Kontrollarm (Dacarbazin) auf 10 Monate signifikant verlängerte [6, 17]: Fortan kam es zu einer hoch dynamischen Entwicklung im Bereich der Checkpointinhibitortherapie mit einer Reihe von Zulassungen bei unterschiedlichsten Entitäten (Übersicht zum aktuellen Zulassungsstatus in - Tab. 1). Beispielhaft seien hier einige Entitäten mit den jeweiligen „Meilensteinarbeiten“" kurz vorgestellt.

\section{Melanom}

Nach dem Einsatz von Ipilimumab zeigten beim malignen Melanom mehrere Studien (CheckMate238 [24], CheckMate067 [25] und KEYNOTE-006 [17]), dass die beiden PD-1-Inhibitoren Nivolumab und Pembrolizumab dem CTLA4-Inhibitor Ipilimumab als Monotherapie bei Patienten mit fortgeschrittenem Melanom signifikant überlegen sind. Die Kombination aus Ipilimumab und Nivolumab erbrachte - wenn auch in den bisherigen Studien ohne statistische Signifikanz - eine weitere Verbesserung.

\section{Nichtkleinzelliges Bronchial- karzinom (NSCLC)}

Die KEYYNOTE-24-Studie wurde als Phase-III-Studie an 305 Patienten mit unbehandeltem fortgeschrittenem NSCLC mit mehr als $50 \%$ Expression von PD-L1 in der Biopsie durchgeführt und verglich Pembrolizumab mit einer platinbasierten Chemotherapie. Hier zeigte sich ein signifikant besseres progressionsfreies 


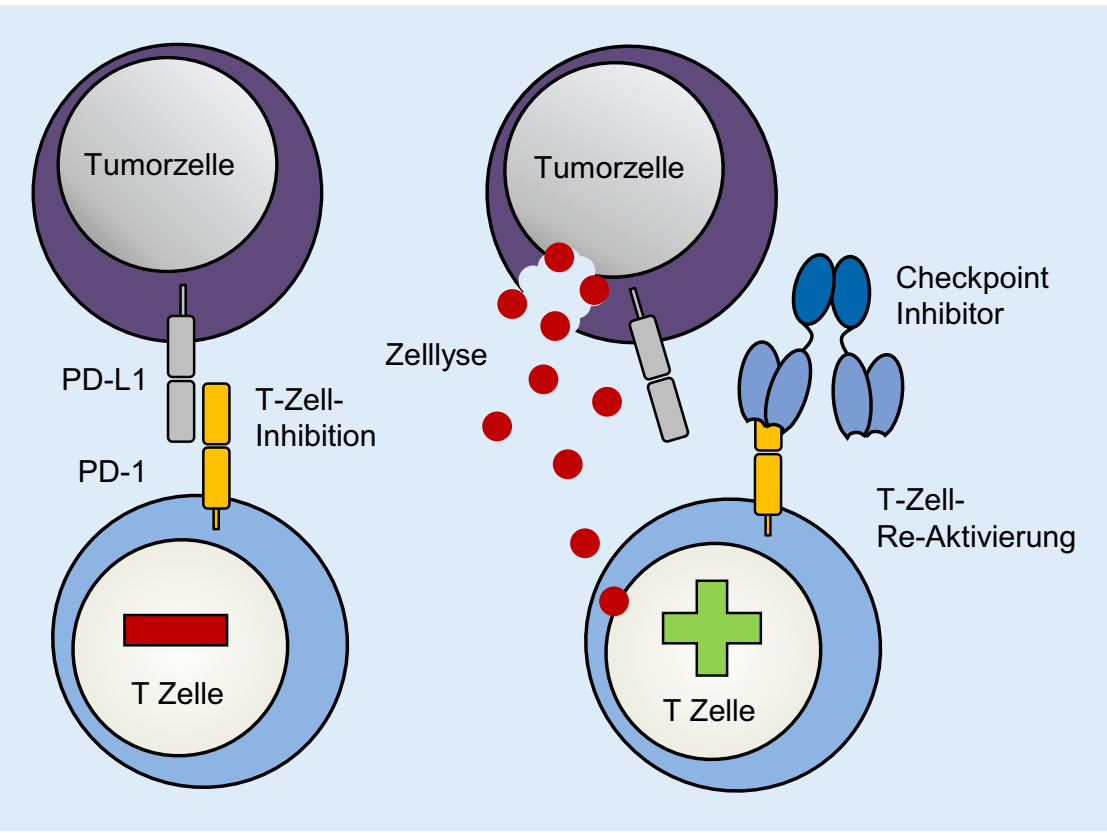

Abb. 1 A Aktivierung von T-Zellen durch Checkpointblockade. Inhibitorische Signalwege (z. B. PD-1/PD-L1) verhindern eine überschießende Immunreaktion nach T-Zell-Aktivierung, die sich auch Tumorzellen zu Nutze machen, um einer Immunantwort zu entgehen. Mit Hilfe monoklonaler Antikörper lassen sich diese Signalwege blockieren, was zu einer Reaktivierung der T-Zell-Antwort führt. PD-1 Programmierter-Zelltod-1-Molekül; PD-L1 Programmierter-Zelltod-1-Ligand

Überleben (PFS) für den Pembrolizumab-Arm (10,3 vs. 6 Monate; HR 0,60, $p=0,005$; [15]). Konsekutiv stellt Pembrolizumab aktuell die Erstlinientherapie der Wahl für Patienten mit NSCLC und Expression von PD-L1 $>50 \%$ dar. Durch die Hinzunahme einer konventionellen Chemotherapie zu Pembrolizumab (KEYNOTE-189 [3]) bzw. Atezolizumab als PD-L1-Blocker (IMpower150 [20]) konnte jüngst gezeigt werden, dass diese Kombinationen einer platinbasierten Chemotherapie auch unabhängig vom PD-L1-Status überlegen sind.

\section{Nierenzellkarzinom}

Bei Patienten mit fortgeschrittenem Nierenzellkarzinom konnte bereits 2015 gezeigt werden, dass nach Vortherapie mit einem Angiogeneseinhibitor eine Therapie mit Nivolumab der Behandlung mit Everolimus überlegen ist [10]. Für die Patienten mit intermediärem oder hohem Risiko-Score zeigte sich jüngst die Kombination aus Nivolumab und Ipilimumab dem bisherigen Erstlinienstandard überlegen (CheckMate214 [11]). Darüber hinaus erbrachten aktuelle Studien positive Ergebnisse für die Kombination aus ICPi und Tyrosinkinaseinhibitor (TKI [16]).

\section{T-Zell-Rezeptor-T-Zellen und chimäre Antigenrezeptor-T- Zellen}

Neben der Aktivierung einer endogenen Immunantwort durch Checkpointinhibitoren stellt der adoptive Transfer von gentechnisch veränderten T-Zellen, welche mit einem tumorantigenspezifischen Rezeptor ausgestattet sind, ein weiteres wirksames und hoffnungsvolles Therapiekonzept in der Krebsimmuntherapie dar. Neben klassischen T-Zell-Rezeptoren (TCR) kommen hierv. a. synthetische Rezeptoren, sog. chimäre Antigenrezeptoren (CAR) zum Einsatz. Ein Vorteil gegenüber der Therapie mit monoklonalen Antikörpern liegt in der Proliferation und möglichen Persistenz von TCR- und CAR-T-Zellen im Patienten.

\section{T-Zell-Rezeptor-T-Zellen}

T-Zell-Rezeptoren erkennen endogen prozessierte Fragmente von Tumorantigenen (Peptide) im Kontext definierter HLA-Antigene. Bereits 2006 zeigte eine erste klinische Studie mit einem MART1-spezifischen TCR die Machbarkeit und Wirksamkeit von TCR-genmodifizierten T-Zellen bei Patienten mit metastasiertem Melanom [9].

Weitere Erfolge konnten mit TCR-TZellen gegen die Tumorantigene MAGEA3, gp 100, CEA und NY-ESO-1 bei Patienten mit hämatologischen und soliden Tumoren erzielt werden. In einigen Studien wurde jedoch auch von erheblichen Toxizitäten berichtet, bei denen TCRT-Zellen niedrig exprimiertes Zielantigen auch auf gesunden Geweben erkannten oder fatale Kreuzreaktivitäten gegen diese induzierten. Aktuell sind weltweit mehr als 100 Studien mit TCR-genmodifizierten T-Zellen verschiedener Spezifitäten bei unterschiedlichen Tumorentitäten registriert. Auch in Deutschland wird derzeit in einer Phase-I/-II-Studie die Sicherheit und Wirksamkeit von PRAME/HLA-A2-spezifischen TCR-TZellen bei Patienten mit rezidivierter/ refraktärer akuter myeloischer Leukämie, myelodysplastischem Syndrom und multiplem Myelom getestet (NCT03503968). 


\section{Chimäre Antigenrezeptor-T-Zellen}

Im Gegensatzzu TCR-T-Zellen erkennen CAR-T-Zellen HLA-unabhängig Antigene auf der Oberfläche von Tumorzellen, was einen breiteren klinischen Einsatz erlaubt. Synthetische CARs bestehen aus einem antigenbindenden variablen Einzelkettenfragment ( $\mathrm{scFv}$, „single chain variable fragment") eines monoklonalen Antikörpers, welcher über eine Transmembrandomäne an eine intrazelluläre signalgebende Domäne des TCR-

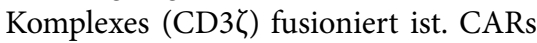
der neueren Generation exprimieren zusätzlich eine oder mehrere kostimulatorische Domänen (•Abb.2), welche die Effektivität, Proliferation und Langlebigkeit der CAR-T-Zellen maßgeblich verbessern.

Das Einbringen des CAR in die T-Zellen der Patienten erfolgt analog zum TCR-Gentransfer mit Hilfe von Retrooder Lentiviren in spezialisierten GMPLaboren und dauert in Abhängigkeit vom Herstellungsprozess ca. 10-14 Tage. Vor T-Zell-Rückgabe erhält der Patient in der Regel eine lymphozytendepletierende Chemotherapie. Nach ersten enttäuschenden klinischen Studien mit CAR-T-Zellen bei soliden Tumoren gelang der Durchbruch der CAR-TTherapie mit CD19-spezifischen CARs der neueren Generation bei Patienten mit fortgeschrittenen B-Zell-Neoplasien. CD19 ist ein Oberflächenprotein, welches von B-Lymphozyten und B-ZellNeoplasien wie der akuten lymphatischen B-Zell-Leukämie (B-ALL) und B-Zell-Non-Hodgkin-Lymphomen (B$\mathrm{NHL}$ ) exprimiert wird.

\section{Zugelassene CAR-T-Zellprodukte}

Die Marktzulassung der ersten beiden CD19-spezifischen CAR-T-Zellprodukte Tisagenlecleucel (Kymriah; Fa. Novartis, Basel, Schweiz) und Axicabtagene Ciloleucel (Yescarta; Fa. Kite Pharma, Santa Monica, CA, USA) im Jahr 2017 in den USA und im August 2018 in Europa begründen die Hoffnung auf eine neue Ära in der Krebstherapie. Die Zulassungen beruhen auf den beeindruckenden Ergebnissen einarmiger multizentrischer Studien an pädiatrischen Patienten mit refraktärer/rezidivierter (r/r) B-ALL (Ti-

Onkologe 2019· 25 (Suppl 1):S77-S82 https://doi.org/10.1007/s00761-019-0609-y

(c) Der/die Autor(en) 2019

\section{Heudobler $\cdot$ W. Herr $\cdot$ S. Thomas \\ Immuntherapien in der Hämatologie und Onkologie}

\section{Zusammenfassung}

Hintergrund. Die moderne Immunonkologie hat die Krebstherapie in den letzten Jahren revolutioniert.

Fragestellung. Diese Arbeit gibt einen Überblick über die aktuellen Therapiemöglichkeiten.

Material und Methoden. Ein Review der aktuellen Studiendaten wurde erstellt. Ergebnisse. In der Onkologie sind Immuncheckpointinhibitoren (ICPi) in der Monotherapie oder auch in Kombination mit anderen Medikamenten zum neuen Therapiestandard bei vielen Entitäten geworden. In der Therapie hämatologischer Neoplasien zeigen CAR-T-Zellen (CAR chimäre
Antigenrezeptoren) und bispezifische Antikörper beeindruckende Ansprechraten. Die Tumorvakzination stellt eine weitere immunologische Therapieform mit hohem Potenzial dar, die jedoch noch keinen breiten Eingang in die Klinik gefunden hat. Schlussfolgerungen. Die Immunonkologie gehört mit unterschiedlichen Ansätzen in vielen Entitäten aktuell zum Therapiestandard.

Schlüsselwörter Immunonkologie - Immuncheckpoint . TCR/CAR-T-Zell-Therapie · Bispezifische Antikörper - Vakzination

\section{Immunotherapies in hematology and oncology}

\section{Abstract}

Background. Modern immuno-oncology has revolutionized cancer therapy in recent years. Objective. This article provides an overview of current treatment options.

Material and methods. Review of the current study situation.

Results. In oncology immune checkpoint inhibitors (ICPi) in monotherapy or also in combination with other drugs have become the standard of care in many tumor entities. In the treatment of hematological neoplasms chimeric antigen receptor (CAR) T-cells and bispecific antibodies have shown impressive response rates. Tumor vaccination represents another treatment option with high potential, but has not yet achieved broad clinical application.

Conclusion. Immuno-oncology with diverse approaches has become the current standard of care in cancer treatment.

\section{Keywords}

Immuno-oncology · Immune checkpoint . TCR/CAR T-cell therapy · Bispecific antibodies . Vaccination sagenlecleucel) und erwachsenen Patienten mit $\mathrm{r} / \mathrm{r}$ diffus großzelligem B-NHL (Tisagenlecleucel und Axicabtagene Ciloleucel; $[12,19])$. In allen 3 Studien zeigte sich die Therapie hoch effektiv mit Ansprechraten, die auf einen deutlichen Vorteil gegenüber historischen Kontrollen hindeuten. Für die Indikation DLBCL lag die Wahrscheinlichkeit, nach einem Jahr am Leben zu sein, bei $49 \%$ (Tisagenlecleucel) und $60 \%$ (Axicabtagene Ciloleucel); Das mediane Gesamtüberleben für Patienten mit kompletter Remission nach CAR-T-Therapie war in beiden Studien noch nicht erreicht. Die Therapie mit CAR-T-Zellen ist mit verschiedenen Nebenwirkungen verbunden. Als häufigste unerwünschte Wirkung (Grad $3 / 4$ ) treten in den ersten 8 Wochen nach der CAR-T-Infusion das Zytokinfreisetzungssyndrom, Enzephalopathien, Zytopenien und Infektionen auf, die sich bei frühzeitiger Therapie in der Regel gut kontrollieren lassen. Essenziell sind eine gute Schulung des Behandlungsteams und eine enge Vernetzung mit Neurologen und Intensivmedizinern.

\section{Weitere Entwicklungen}

Neben dem Erfolg der CAR-T-Zell-Therapie bei CD19-positiven B-Zell-Neoplasien gibt es vielversprechende Ergebnisse mit CAR-T-Zellen (bb2121) mit Spezifität für das B-Zell-Reifungsantigen („Bcell maturation antigen“; BCMA) bei $\mathrm{Pa}$ tienten mit stark vorbehandelten $\mathrm{r} / \mathrm{r}$ multiplen Myelomen [14]. Eine Vielzahl weiterer klinischer Studien (weltweit >200 


\section{Tab. 1 Aktueller Zulassungsstatus von Immuncheckpointinhibitoren}

\begin{tabular}{|c|c|c|c|}
\hline $\begin{array}{l}\text { Zulassungs- } \\
\text { status }\end{array}$ & Wirkstoff & $\begin{array}{l}\text { Ziel- } \\
\text { struktur }\end{array}$ & Indikation \\
\hline \multirow{6}{*}{$\begin{array}{l}\text { In der EU } \\
\text { zugelassen }\end{array}$} & Ipilimumab & CTLA-4 & Melanom, Nirenzellkarzinom \\
\hline & Nivolumab & PD-1 & $\begin{array}{l}\text { Melanom, NSCLC, Nierenzellkarzinom, klassi- } \\
\text { sches Hodgkin-Lymphom, Plattenepithelkarzi- } \\
\text { nome des Kopf-Hals-Bereichs, Urothelkarzinom }\end{array}$ \\
\hline & Pembrolizumab & PD-1 & $\begin{array}{l}\text { Melanom, NSCLC, klassisches Hodgkin-Lym- } \\
\text { phom, Plattenepithelkarzinome des Kopf-Hals- } \\
\text { Bereichs, Urothelkarzinom }\end{array}$ \\
\hline & Avelumab & PD-L1 & Merkel-Zell-Karzinom \\
\hline & Atezolizumab & PD-L1 & NSCLC, Urothelkarzinom \\
\hline & Durvalumab & PD-L1 & NSCLC \\
\hline \multirow[t]{4}{*}{$\begin{array}{l}\text { In den USA } \\
\text { zugelassen }\end{array}$} & Pembrolizumab & PD-1 & $\begin{array}{l}\text { Zusätzlich: bei Mikrosatelliteninstabilität (unab- } \\
\text { hängig von der Histologie) }\end{array}$ \\
\hline & Atezolizumab & PD-L1 & Zusätzlich: TNBC, SCLC \\
\hline & Durvalumab & PD-L1 & Zusätzlich: Urothelkarzinom \\
\hline & Cemiplimab & PD-1 & Plattenepithelkarzinom \\
\hline
\end{tabular}

Studien) laufen derzeit bei sowohl hämatologischen als auch soliden Neoplasien. Ein zentrales Problem nach CART-Zell-Therapie ist die Entwicklung von Rezidiven durch den Verlust des Zielantigens auf der Tumorzelle (z. B. CD19Verlust beim B-NHL). Um dies zu vermeiden, zielen neue Strategien darauf $a b$, CAR-T-Zellen mit bispezifischen CARMolekülen auszustatten, die gleichzeitig 2 Antigene (z. B. CD19 und CD20) aufder Tumorzelle erkennen und somit die antitumorale Wirksamkeit verstärken. Weitere Ansätze, die Effektivität von CART-Zellen zu verbessern, zielen auf die Kombination mit anderen Immuntherapien (ICPi oder Vakzinierung) sowie das Einbringen zusätzlicher kostimulatorischer Moleküle oder die Herstellung von zytokinsezernierenden CAR-T-Zellen. Bei diesen sog. Next-GenerationCAR-T-Zell-Therapien besteht die Hoffnung, auch bei soliden Tumoren - die bislang nur sehr begrenzt auf CAR-TTherapien ansprechen - Remissionen $\mathrm{zu}$ erzielen [1]. Um den komplexen Herstellungsprozess von autologen CAR-T-Präparaten zu erleichtern und die Therapie für eine größere Patientenpopulation verfügbar zu machen, wird derzeit auch die Effektivität von universell einsetzbaren allogenen CAR-T-Zellen in klinischen Studien untersucht, bei denen die natürlich vorkommenden TCR-Gene mit
Hilfe von Genomeditierungstechnologie gezielt ausgeschaltet werden [13].

\section{Bispezifische Antikörper}

Bispezifische Antikörper (bsAbs) kombinieren die Antigenbindungsstellen von 2 monoklonalen Antikörpern in einem Molekül. Die enge Nachbarschaft aus tumorspezifischem Antigen und EffektorT-Zelle resultiert so in einer gezielten Aktivierung der T-Zelle. Der Mechanismus ähnelt damit dem von CAR-T-Zellen. Gemeinsam stellen CAR-T-Zellen und bsAbs die wirksamsten Werkzeuge für HLA-unabhängige T-Zell-Antwort gegen Tumorzellen dar, wobei im Gegensatz zu CAR-T-Zellen bsAbs „off the shelf" einsetzbar und damit schneller verfügbar sind. Demgegenüber ist ihre Wirksamkeit aber von einer prolongierten Applikation abhängig [22].

Unter den unterschiedlichen Formaten von bsAbs, die entwickelt wurden, sind bispezifische T-Zell-Engager (BiTEs) klinisch aktuell von besonderem Interesse. BiTEs bestehen aus 2 Einzelketten aus variablen Fragmenten, die spezifisch für CD3 sowie das jeweilige Tumorantigen sind (•Abb. 3). Der CD19-spezifische BiTE Blinatumomab zeigte ein beeindruckendes Ansprechen bei Patienten mit stark vorbehandelter B-ALL. Das mOS lag bei 7,7 Mona- ten für Blinatumomab vs. 4 Monaten für die Chemotherapie $(p=0,01$; [7]). Aktuell sind etliche bsAbs in Entwicklung und klinischer Erprobung. Designtechnisch werden hierbei neben BiTEs auch DARTS („dual affinity retargeting“) oder DuoBody $^{\circledR}$ angewendet. Zielstrukturen stellen neben CD19 auch CD20, BCMA, CD138 oder auch CD3 und CD123 dar, sodass neben den B-ZellNeoplasien auch das multiple Myelom sowie andere myeloische Neoplasien in den Behandlungsfokus rücken.

\section{Vakzination mit tumorassoziierten Antigenen}

Neben den genannten Möglichkeiten stellt die Vakzinierung mit tumorassoziierten Antigenen eine weitere Therapieform dar. Eine solche aktive Immunisierung zielt auf die Überwindung der Ignoranz des Immunsystems gegenüber dem im Patienten vorhandenen Tumor. Voraussetzung für diese Therapie ist die Kenntnis geeigneter tumorassoziierter Antigene(TAA; [4]). In klinischen PhaseI-/-II-Studien, in denen Tumorpatienten im fortgeschrittenen Krankheitsstadium mit einzelnen TAA vakziniert wurden, zeigte sich generell eine gute Verträglichkeit, und es gab einzelne partielle und auch komplette Tumorremissionen. Ein Beispiel für eine Vakzination ist die Impfung mit dem Idiotyp-Immunglobulin bei Patienten mit B-ZellLymphomen, die in einigen Fällen zu deutlichen Tumorremissionen führte [21]. Ein Nachteil einer Vakzination mit Tumorneoantigenen ist, dass ihr Auftreten zumeist individualspezifisch ist und damit in der Regel aufwendige Vorarbeiten zur Antigencharakterisierung und personalisierten Vakzineherstellung notwendig macht.

\section{》) Die Herstellung persona- lisierter Tumorvakzinen beim metastasierten Melanom und Glioblastom ist möglich}

Allerdings haben jüngere Arbeiten beim metastasierten Melanom und Glioblastom mit Hilfe neuer Technologien die 
1. Generation

2. Generation

3. Generation

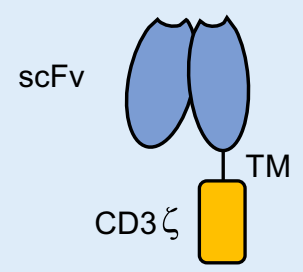

CD28/4-1BB
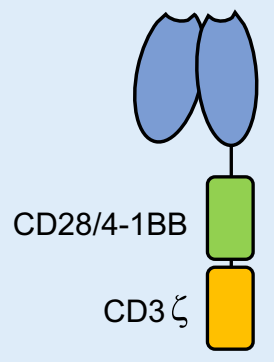

4-1BB/OX-40

$\mathrm{CD} 3 \zeta$

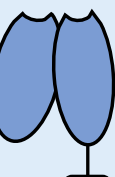

CD28

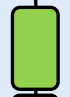

$3 \zeta$

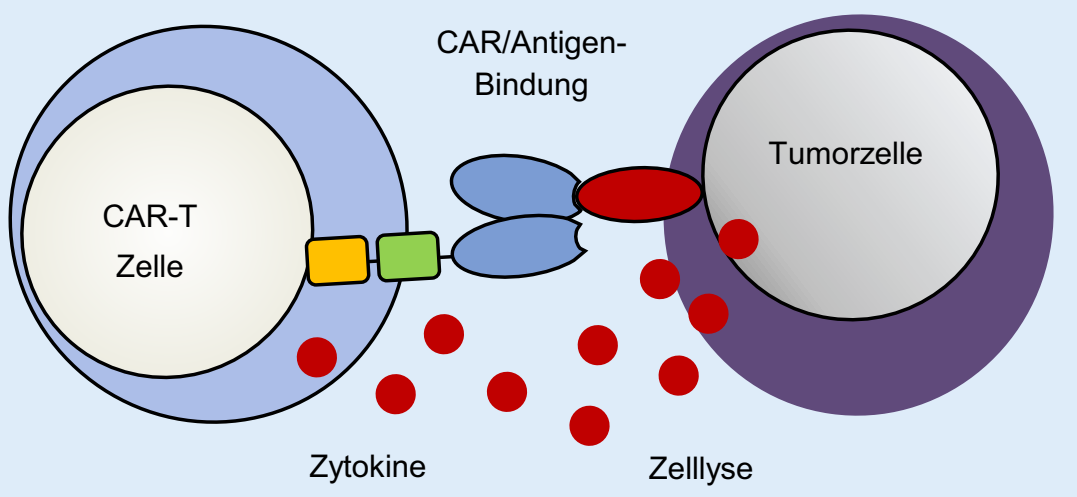

Abb. 2 \ Aufbau von chimären Antigenrezeptoren (CARs). Zur Herstellung von CARs werden die extrazellulären antigenbindenen Domänen eines monoklonalen Antikörpers als Einzelkettenfragement (scFv) über eine kurze Gelenk- und Transmembrandomäne (TM) an signalgebende Domänen des TCRKomplexes fusioniert. CARs der 1. Generation enthalten eine CD3 2. oder 3. Generation zusätzlich zu CD3 eine oder zwei weitere kostimulatorische Signaldomänen enthalten, die die Effekte der CAR-T-Zellen verstärken. Nach Antigenbindung kommt es durch die Auslösung einer Signalkaskade innerhalb der CAR-T-Zelle zu einer Immunantwort in Form einer direkten zytotoxischen Lyse der Tumorzelle durch die CAR-T-Zelle und zur Ausschüttung von Zytokinen, die eine indirekte Immunantwort über die Aktivierung von weiteren Immunzellen auslöst

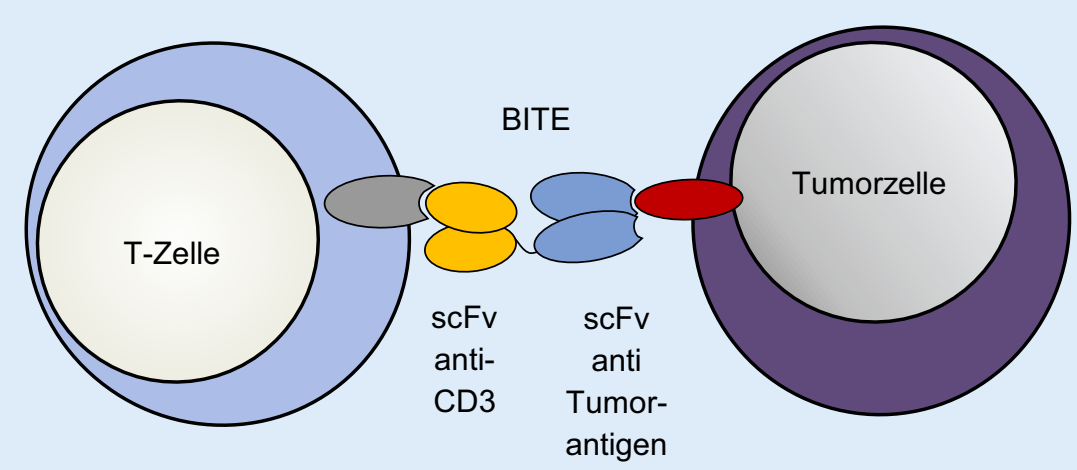

Abb. 3 Aufbau eines bsAbs am Beispiel eines BiTE. BiTEs bestehen aus 2 scFv-Fragmenten, die über einen Peptidlinker miteinander verbunden sind. Eines der beiden scFv weist eine Spezifität für CD3, das andere eine für ein definiertes Tumorantigen (z. B. CD19) auf. Durch Bindung an die Zielantigene führen BiTEs zu einer Verlinkung von T-Zelle und Tumorzelle, die eine spezifische T-Zell-Antwort induziert. bsAbs bispezifische Antikörper, BiTE bispezifischer T-Zell-Engager, $s C F v$ "single chain variable fragment"
Machbarkeit der Herstellung personalisierter Tumorvakzinen gezeigt $[5,18]$. Bei einigen Patienten wurden unter Vakzination signifikante Tumorrückbildungen beobachtet.

Die erste klinische Phase-III-Studie (IMPACT), die zur vorübergehenden $\mathrm{Zu}$ lassung (Food and Drug Administration 2010) einer Impftherapie bei bestehender metastasierter Tumorerkrankung geführt hat, wurde bei insgesamt 512 Patienten mit Prostatakarzinom durchgeführt [8]: Patienten, die die zelluläre Vakzine Sipuleucel-T (bestehend aus autologen dendritischen Zellen ex vivo beladen mit einem Fusionsprotein aus prostataspezifischer saurer Phosphatase und Granulozyten-Makrophagen-Kolonie-stimulierendem Faktor) erhielten, hatten ein um 4,1 Monate verbessertes Überleben, obwohl es keinen Unterschied im progressionsfreien Überleben im Vergleich zum Kontrollarm gab, ein Phänomen, das man häufiger bei Immuntherapien beobachtet.

\section{Fazit für die Praxis}

- Immuncheckpointinhibitoren haben die Krebstherapie revolutioniert und stellen in Mono- oder Kombinationstherapien bei vielen Entitäten den neuen Therapiestandard dar.

- CAR-T-Zellen (CAR chimäre Antigenrezeptoren) induzieren insbesondere bei hämatologischen Neoplasien beeindruckende Ansprechraten. Herstellprozess und Therapieablauf sind komplex und bedürfen einer guten Koordination und Schulung des gesamten Behandlungsteams.

- Bislang ist die Wirksamkeit von CART-Zellen bei soliden Tumoren sehr begrenzt. Es besteht jedoch die Hoffnung, mit neuen Entwicklungen die Wirksamkeit von CAR-T-ZellTherapien bei soliden Tumoren zu optimieren.

- Bispezifische Antikörper aktivieren T-Zellen in räumlicher Nähe zur Tumorzelle und lösen so eine Immunantwort aus. Insbesondere bei der akuten lymphatischen B-ZellLeukämie (B-ALL) zeigen sich beeindruckende Ansprechraten. 


\section{- Die Vakzination mit tumorassozi- ierten Antigenen ist gut verträglich und induziert teilweise signifikante Remissionen.}

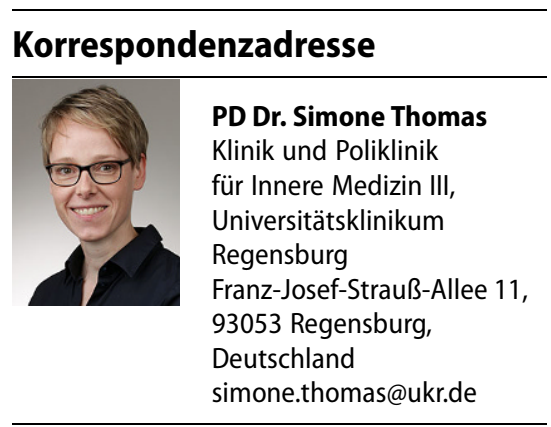

\section{Einhaltung ethischer Richtlinien}

Interessenkonflikt. D. Heudobler: Vortragshonorare, Beratertätigkeit und Reisekostenübernahmen:BMS, Celgene, MSD, Pfizer, Roche. W. Herr: Vortragshonorare, Beratertätigkeit und Reisekostenübernahmen: Amgen, BMS, Celgene, MSD, Novartis, Pfizer, Roche. S. Thomas: Vortragshonorare, Beratertätigkeit und Reisekostenübernahmen: Amgen, Celgene, Gilead, Medigene AG, Novartis, Pfizer.

Für diesen Beitrag wurden von den Autoren keine Studien an Menschen oder Tieren durchgeführt. Für die aufgeführten Studien gelten die jeweils dort angegebenen ethischen Richtlinien.

Open Access. Dieser Artikel wird unter der Creative Commons Namensnennung 4.0 International Lizenz (http://creativecommons.org/licenses/by/4.0/deed. de) veröffentlicht, welche die Nutzung, Vervielfältigung, Bearbeitung, Verbreitung und Wiedergabe in jeglichem Medium und Format erlaubt, sofern Sie den/die ursprünglichen Autor(en) und die Quelle ordnungsgemäßnennen, einen Link zur Creative Commons Lizenz beifügen und angeben, ob Änderungen vorgenommen wurden.

\section{Literatur}

1. Abken H (2017) Driving CARs on the Highway to Solid Cancer: Some Considerations on the Adoptive Therapy with CART Cells. Hum Gene Ther 28(11):1047-1060

2. Cristescu R, Mogg R, Ayers M et al (2018) Pantumor genomic biomarkers for PD-1 checkpoint blockade-based immunotherapy. Science. https:// doi.org/10.1126/science.aar3593

3. Gandhi L, Rodríguez-Abreu D, Gadgeel $S$ et al (2018) Pembrolizumab plus Chemotherapy in Metastatic Non-Small-Cell Lung Cancer. N Engl J Med 378(22):2078-2092

4. Haen SP, Rammensee H-G (2013) The repertoire of human tumor-associated epitopes-identification and selection of antigens and their application in clinical trials. Curr Opin Immunol 25(2):277-283

5. Hilf N, Kuttruff-Coqui S, Frenzel K et al (2019) Actively personalized vaccination trial for newly diagnosed glioblastoma. Nature 565(7738):240-245
6. Hodi FS, O'Day SJ, McDermott DF et al (2010) Improved survival with ipilimumab in patients with metastatic melanoma. $\mathrm{N}$ Engl J Med 363(8):711-723

7. Kantarjian H, Stein A, Gökbuget N et al (2017) Blinatumomab versus Chemotherapy for Advanced Acute Lymphoblastic Leukemia. N Engl J Med 376(9):836-847

8. Kantoff PW, Higano CS, Shore ND et al (2010) Sipuleucel-T immunotherapy for castration-resistant prostate cancer. NEngl J Med 363(5):411-422

9. Morgan RA, Dudley ME, Wunderlich JR et al (2006) Cancer regression in patients after transfer of genetically engineered lymphocytes. Science 314(5796):126-129

10. Motzer RJ, Escudier B, McDermott DF et al (2015) Nivolumab versus Everolimus in Advanced RenalCell Carcinoma. N Engl J Med 373(19):1803-1813

11. Motzer RJ, Tannir NM, McDermott DF et al (2018) Nivolumab plus Ipilimumab versus Sunitinib in Advanced Renal-Cell Carcinoma. N Engl J Med 378(14):1277-1290

12. Neelapu SS, Locke FL, Bartlett NL et al (2017) Axicabtagene Ciloleucel CAR T-Cell Therapy in Refractory Large B-Cell Lymphoma. N Engl J Med 377(26):2531-2544

13. Qasim W, Zhan H, Samarasinghe S et al (2017) Molecular remission of infant B-ALL after infusion of universal TALEN gene-edited CAR T cells. Sci Transl Med. https://doi.org/10.1126/scitranslmed. aaj2013

14. Raje N, Berdeja J, Lin Y et al (2019) AntiBCMA CAR T-Cell Therapy bb2121 in Relapsed or Refractory Multiple Myeloma. N Engl J Med 380(18):1726-1737

15. Reck M, Rodríguez-Abreu D, Robinson AG et al (2016) Pembrolizumab versus Chemotherapy for PD-L1-Positive Non-Small-Cell Lung Cancer. N Engl JMed 375(19):1823-1833

16. Rini Bl, Plimack ER, Stus V et al (2019) Pembrolizumab plus Axitinib versus Sunitinib for Advanced Renal-Cell Carcinoma. N Engl J Med 380(12):1116-1127

17. Robert C, Thomas L, Bondarenko I et al (2011) Ipilimumab plus dacarbazine for previously untreated metastatic melanoma. N Engl J Med 364(26):2517-2526

18. Sahin U, Derhovanessian E, Miller M et al (2017) Personalized RNA mutanome vaccines mobilize poly-specific therapeuticimmunityagainst cancer. Nature 547(7662):222-226

19. Schuster SJ, Bishop MR, Tam CS et al (2019) Tisagenlecleucel in Adult Relapsed or Refractory Diffuse Large B-Cell Lymphoma. N Engl J Med 380(1):45-56

20. Socinski M A, Jotte R M, Cappuzzo F et al (2018) Overall survival (OS) analysis of IMpower150, a randomized $\mathrm{Ph} 3$ study of atezolizumab (atezo) + chemotherapy (chemo) \pm bevacizumab (bev) v chemo + bev in $1 \mathrm{~L}$ nonsquamous (NSQ) NSCLC. JCO36(15_suppl): 9002

21. Timmerman JM, Czerwinski DK, Davis TA et al (2002) Idiotype-pulsed dendritic cell vaccination for B-cell lymphoma: clinical and immune responses in 35 patients. Blood 99(5):1517-1526

22. Velasquez MP, Bonifant CL, Gottschalk S (2018) Redirecting $\mathrm{T}$ cells to hematological malignancies with bispecific antibodies. Blood 131(1):30-38

23. Walker LSK, Sansom DM (2011) The emerging role of CTLA4 as a cell-extrinsic regulator of T cell responses. Nat Rev Immunol 11(12):852-863

24. Weber J, Mandala $M$, Del Vecchio $M$ et al (2017) Adjuvant Nivolumab versus Ipilimumab in
Resected Stage III or IV Melanoma. N Engl J Med 377(19):1824-1835

25. Wolchok JD, Chiarion-Sileni V, Gonzalez R et al (2017) Overall Survival with Combined Nivolumab and Ipilimumab in Advanced Melanoma. N Engl J Med 377(14):1345-1356 